\title{
Modeling individual variation in visual search with reinforcement learning
}

\author{
Ben Lonnqvist ${ }^{1 \diamond}$, Micha Elsner ${ }^{2{ }^{*}}$, Amelia R. Hunt ${ }^{3}$, Alasdair D. F. Clarke ${ }^{4}$
}

1 Brain Mind Institute, École Polytechnique Fédérale de Lausanne (EPFL), Switzerland

2 Department of Linguistics, Ohio State University, Columbus, Ohio, USA

3 School of Psychology, University of Aberdeen, Aberdeen, UK

4 Department of Psychology, University of Essex, Colchester, UK

(2These authors contributed equally to this work.

* melsner0@gmail.com

\begin{abstract}
Experiments on the efficiency of human search sometimes reveal large differences between individual participants. We argue that reward-driven task-specific learning may account for some of this variation. In a computational reinforcement learning model of this process, a wide variety of strategies emerge, despite all simulated participants having the same visual acuity. We conduct a visual search experiment, and replicate previous findings that participant preferences about where to search are highly varied, with a distribution comparable to the simulated results. Thus, task-specific learning is an under-explored mechanism by which large inter-participant differences can arise.
\end{abstract}

\section{Introduction}

Visual search is integral to human behaviour and its study lies at the intersection of vision science and more general cognitive psychology. Performance in visual search is determined by basic visual and motor properties (like the salience of the target against the distractors, and how the eye moves), and higher-level strategies (for a review, see (9)), and is generally studied using a paradigm in which an observer is shown a stimulus consisting of collection of discrete search items. The observer is tasked with responding, as quickly and as accurately as possible, whether the target is present or absent. Although it is well-established that attention can shift in the absence of eye movements (e.g. (36)), in the current study we focus on what is often referred to as "overt" attention, that is, the sequence of fixations participants make to find a target during search.

One strand of research in this field has been the question of the extent to which human observers can execute an optimal sequence of eye-movements during visual search. While an ideal observer (30) model offers a good explanation for human search behaviour in some contexts, large departures from optimality have often been observed. For example, (29) used a task in which observers were required to fixate one of two potential target locations, or half way between them. An eye tracker was used to monitor their saccades, and upon fixation, a small target would appear at one of the two locations. As the target is difficult to see using peripheral vision, the choice of where to look depends on the separation between the two potential locations. When they are close to one another, the optimal fixation location is the midpoint between 

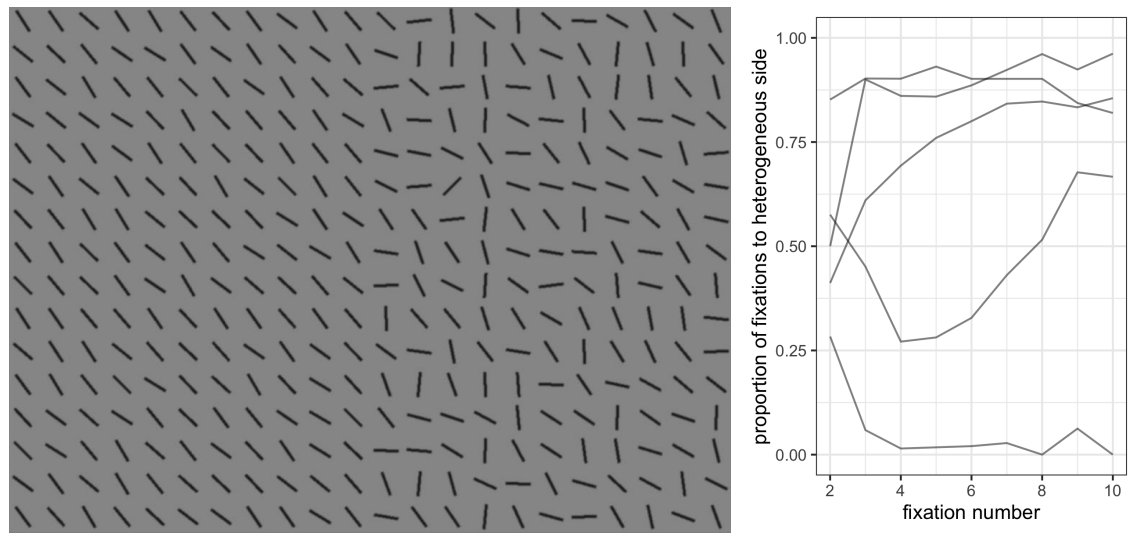

Fig 1. (left) Example of the split-half visual search stimulus. The target is 6-down, 9 from the right. (right). Results from five participants from (8) .

them, as this will allow the observer to detect the target at either location. If the separation distance is greater, observers can maximise their expected accuracy by fixating one of the two targets, increasing their chance of detection should the target appear there, at the expense of the other location. Perhaps surprisingly, the participants in this study did not follow this behaviour, and in fact, their choice of where to fixate appeared to be completely independent of the distance between target locations. This result has since been replicated by (7) and both studies show that participants do not, in general, choose an optimal fixation strategy.

In this work, we focus on the "split-half" search task introduced by Nowakowska et al. (34). In this task, participants search for a fixed target (a slanted line) in an array of distractors, as shown in Figure 1. In one half of the array, the distractors are homogeneous and highly dissimilar to the target, causing the target to be more easily visible if it is present; the other half of the array is heterogeneous and must be searched more closely. Nowakowska et al. (34) argue that the optimal search strategy is to fixate only the heterogeneous side of the array, but show that the average proportion of these fixations is about $50 \%$. This average tendency is compatible with simple accounts in which visual search is governed by a random walk (7), but such a model is not a good fit for the behavior of individual participants. In fact, participant behavior is highly variable - some participants do approximate the optimal strategy, searching the heterogeneous side almost exclusively, while others are anti-optimal, preferring to fixate on the homogeneous side.

The cause of these differences in strategy is yet to be determined. Related work on links between individual differences in visual search and personality measures has met with limited success (15, 17). Here we use computational methods to investigate the hypothesis that these differences are driven by the variability inherent in learning from experience.

We present a computational model of the process by which participants learn their strategies for task-specific visual search. We show that implicit learning by a reinforcement learning algorithm can lead to both near-optimal and anti-optimal behavior in this task. These differences can arise purely as a result of variation in learning trajectories, without the necessity of differences in low-level visual acuity. This is because reinforcement learning involves a trade-off between "exploration" of a diverse set of strategies and "exploitation" of the current best strategy to derive good results; learners who focus on exploitation have highly variable learning outcomes, dependent on initial conditions and different experiences during training. We argue that this explains much of the variation between individuals in the split-half visual search task. Learners 
are dependent on their reward signal to learn to find the target, but because the target is hard to find using an unstructured strategy, some learners experience little reward from searching the heterogeneous side. This slows their acquisition of the optimal strategy.

Beyond its application to the array task, our model offers an intermediate between optimal, but computationally demanding optimal models of visual search (30) and fixed, heuristic ones (7). Like optimal models, it assumes that visual search is goal-driven and responsive to characteristics of the stimulus. However, it is computationally inexpensive and thus presents a cognitively plausible account of how human visual searchers can execute near-optimal search strategies without exhaustive computation.

In particular, we present experimental data showing individual variability in the split-half array paradigm. We present a neural network model which learns strategies for visual search. We show that, while this model tends to eventually converge to the optimal seach strategy on the split-half array paradigm, convergence rates vary widely across individual runs, partly due to the influence of the initial confidence required to reach a decision. We argue that these differences in learning trajectories could explain some of the individual variation observed in many visual search paradigms.

Our learning model builds on the same foundation as Najemnik and Geisler (30); it assumes that the viewer mentally represents the scene by keeping track of a posterior distribution over the item at every location. Each fixation provides new information about every object (although the information is more reliable for objects closer to the focal point), and the posterior is updated by Bayes rule to integrate this incoming information with what was already known. The location of the next fixation is then determined based on the posterior. In our model, instead of choosing optimally, the viewer uses a convolutional neural network to pick the next fixation point. This neural network represents a particular search strategy, which can encode preferences for heuristics like looking at likely targets, searching under-explored regions of the grid, and focusing attention on the heterogeneous part of the array.

\section{Model overview}

Reinforcement learning is a family of implicit learning algorithms (40) which iteratively adjust a problem-solving strategy based on the results obtained. These models seek to learn a policy, a mechanism for selecting an action given an observed world state. The quality of a series of actions is determined by an external reward signal which indicates success or failure at solving the problem.

In realistically large problems, policies cannot directly map each observation to an action due to the large number of potential observations. Instead, they must construct some intermediate representation; deep neural networks are a popular and effective mechanism for doing so (28, 38). We follow Mnih et al. (27) in adopting the REINFORCE algorithm (44), a simple and widely used algorithm for reinforcement learning using a neural network.

Mnih et al. (27) learns a neural network which controls a visual sensor and makes simulated saccades. Their system learns to play video games, using a perceptual system composed of a steerable, high-resolution "glimpse network" and a low-resolution network for peripheral vision. Ba et al. (1) applies the same mechanism to the task of recognizing house numbers. These systems, however, are not cognitive models but computationally efficient solutions for image processing; they do not use psychophysical detection functions nor attempt to match human behavior.

Vasilyev (42) explicitly attempts to use reinforcement learning to induce a cognitively plausible model of human visual search. Vasilyev does use a psychophysical detection function (although a simpler one than ours since it depends solely on eccentricity) and does compare the outcome of learning with human behavior. Our study differs from his in its emphasis on individual variation - building on Vasilyev's finding that 
reinforcement learning can generate human-like behavior on average, we also show that it can generate a human-like distribution of search patterns among individuals.

\section{Cognition and reinforcement}

Reinforcement algorithms were originally inspired by cognitive work on animal learning mechanisms following the "conditioned reflex" theory of Pavlov (35), and are still considered to be good high-level models of the learning process; low-level neural mechanisms for reward and reinforcement have also been identified in the brain (37; 26; 40, ch.14,15).

Daw et al. (10) suggests that learning in the brain is carried out by two different cortical mechanisms, corresponding to different types of computational learner. One, a "model-free" learner associated with dopamine reward signals in the striatum, carries out implicit learning, in which actions are directly associated with rewards, without attempting to predict their outcomes. This mechanism is responsible for "habits" which can be slow to adapt to new conditions. The other mechanism, a "model-based" learner associated with the prefrontal cortex, learns by predicting the results of actions on the world, then selecting desired outcomes. This mechanism can adapt more rapidly when the agent's desires or circumstances change. This theory is further supported by evidence from fMRI of subjects involved in sequential decision-making (12).

The algorithms we explore in this paper are model-free, choosing actions based on their reward without attempting to predict their outcome. Under this assumption, we can account for why humans do not always apply the optimal strategy despite its seeming obviousness. If participants applied an explicit model of the world and their own visual systems to predict what a fixation in the heterogeneous array might reveal, it would be obvious that continued looking to the homogeneous side could not result in a target detection. But model-free learning is controlled only by the reward signal, which does not always provide a clear indication that this is the case. This difference in mechanisms fits into the broad distinction between System 1 (fast, implicit decision-making) and System 2 (slow, reasoned planning) outlined by Kahneman (20) as a high-level explanation for a variety of common, non-optimal outcomes in decision-making experiments.

\section{Other models for fixation prediction}

A variety of computational models have been proposed to predict eye movements under various circumstances, some more comparable to this experiment and some less so.

One influential line of research, visual salience modeling, seeks to predict where observers will look in an image when they are not deliberately searching for a particular object. Computational salience modeling, pioneered by Itti et al. (19), now often employs neural network models (5; 43). While these models can be applied to visual search tasks (18), in such cases, they contribute to prediction of eye movements by accounting for viewing biases based only on the scene, independent of the target. While researchers have build on these models by adding top-down task information (e.g., (32, 33, 48) ) such models have not been widely used to model visual search.

Another line of work, beginning with the Feature Integration model (41) and extended by the Guided Search model (45, 47, 46), does seek to explain fixation behavior which depends on the target. Unlike salience-based models, Guided Search assumes that fixations are directed to locations in the image which share some visual features with the target. Like the model proposed here, Guided Search assumes that representations at each point in the display are updated in parallel as more visual information is obtained; this information guides the serial process of attending, or fixating, on different points in the image. Guided Search provides an appealing account 
of how the visual system integrates, or fails to integrate, different types of information about the likely contents of a particular region of the image and its perceived similarity to the target. But its account of visual control assumes that the viewer will attend most to the most "activated" region of the image (46) - in the terminology used by Najemnik and Geisler (30), it is a particular kind of Maximum a Posteriori (MAP) search algorithm. Najemnik and Geisler (30) demonstrate that MAP algorithms do not satisfactorily describe human search behavior.

Thus, Guided Search provides a detailed account of how target detection works in the human visual system. In contrast, we abstract away from this process, estimating detection probabilities from a psychophysical experiment without attempting to model the underlying feature-based processes from which they derive. On the other hand, we provide an account of how strategies for visual control can be learned in novel settings, rather than assuming a fixed strategy of always looking toward the most likely target location.

Finally, we have already discussed Najemnik and Geisler (30), which describes a computationally expensive, but information-theoretically optimal, model for selecting the next fixation point. Najemnik and Geisler (31) provides a computationally much simpler approximation to the optimal procedure with qualitatively similar behavior. Like our own model, the approximation is computed by a convolution over the posterior detection probabilities, an operation which can be computed quickly and partly in parallel. While the approximation offers an appealing account of optimal behavior in visual search tasks, it is not clear how it explains non-optimal or variable behavior. We believe that this kind of cognitively plausible, high-performing strategy should be thought of, not as an inborn and universal part of human cognition, but as the likely outcome of a learning process applied to typical environmental stimuli. In this, it would be comparable to the low-level organization of the visual cortex, which is not entirely innate, but develops based on early exposure to the environment (3; 14).

\section{Results}

\section{Overview}

In the following sections, we present the empirical work underlying our modeling, as well as the results of our modeling simulations. We begin by presenting a target detection experiment, intended to determine how accurately participants in the split-half experiment can determine the orientations of line segments given eccentricity and direction to the fixation point. We show that it is possible to estimate reasonable visibility parameters for use in simulations of visual search.

We then present the results of our computational search simulations, in which the derived visibility map is used to determine what the simulated viewer can determine about the scene at each fixation location. Our simulated viewers use reinforcement learning algorithms to discover policies for searching split-half arrays. We compare simulated to human results in terms of the distribution of strategies, and the rates at which different strategies change over time.

Finally, we show that our algorithm is capable of learning some of the tendencies displayed by the optimal model of (30) on their stimuli, although convergence to this solution is a rare event. We discuss the implications of this finding with respect to learning mechanisms in the human visual system. 

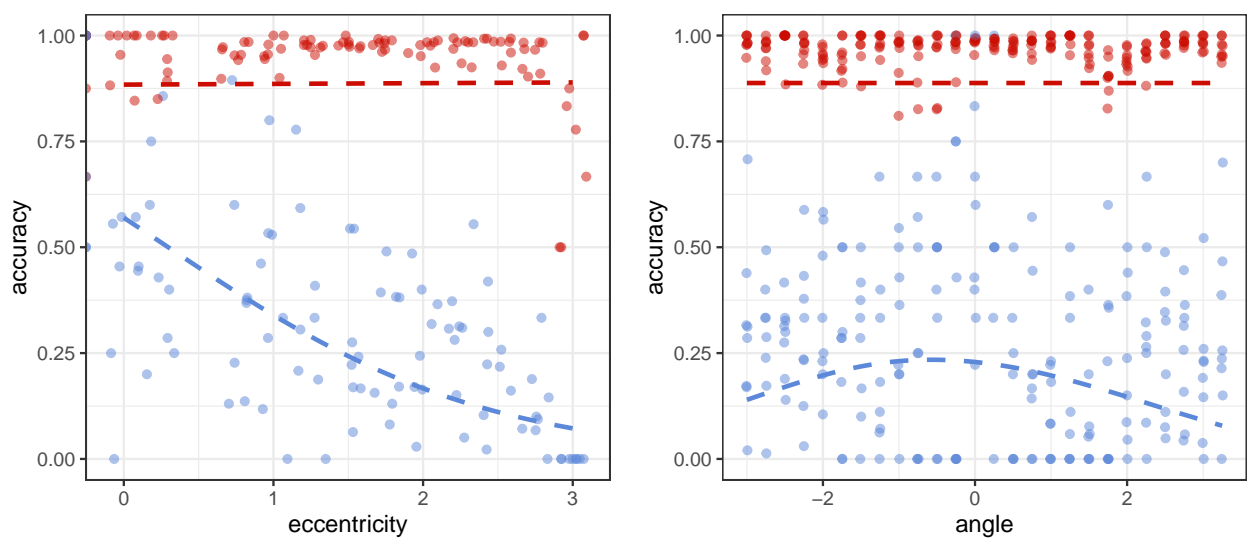

Fig 2. Empirical detection results showing the effect of eccentricity and direction on detection accuracy. Dots show binned average accuracy for each person, while the dotted line shows the performance of our visibility map. Blue $=$ heterogeneous, Red $=$ homogeneous.

\section{Modeling the detection data}

The Detection Experiment was completed by eight observers, each carrying out 2600 trials. On each trial, a stimulus was displayed for $200 \mathrm{~ms}$ and observers were asked to judge whether the target was absent or present. From the results shown in Figure 2 we can see that accuracy was typically close to ceiling for targets against homogeneous distractors, and much worse when surrounded by heterogeneous distractors. We can also clearly see that when the target is on the heterogeneous half of the stimulus, accuracy is dependant on both distance from fixation (eccentricity) and the direction.

One of the difficulties of modelling these data is determining how to deal with false positives. The signal-detection theory framework used by $(\underline{30})$ requires an estimate of the probability of a false positive at each location, relative to the fixation point. In order to estimate this probability, Najemnik \& Geisler carried out an experiment in which the target location was blocked (i.e, observers knew ahead of time where the target could potentially appear). Given well documented covert attention effects, we decided against using this paradigm - telling participants that the target was in one of only two locations would focus their attention, leading to results that do not accurately model the real process of visual search. In other related work, (7) used a simple model that modelled the probability of detecting a target given its location. However, this approach is overly simplistic as it assumes there are no false positives. We can clearly see from Figure 3 that the false positive rate is substantially below ceiling performance.

In our Detection Experiment, the target may appear at any location within the search array. Estimating the probability of false positives in this setting requires a slightly more complex modeling setup. We define a generative model which gives distribution over a participant's response given the scene, then optimize its parameters using a gradient method. We can see from Figures 2 and 3 that our visibility map does a good job of qualitatively matching the behaviour seen in the empirical data and therefore suitable for use in simulations of visual search.

\section{Modeling the Search Data}

We construct reinforcement learning agents using the REINFORCE policy gradient algorithm (44), to learn parameters for convolutional neural networks (24). As summarized above, REINFORCE is a cognitively plausible model of one type of implicit 

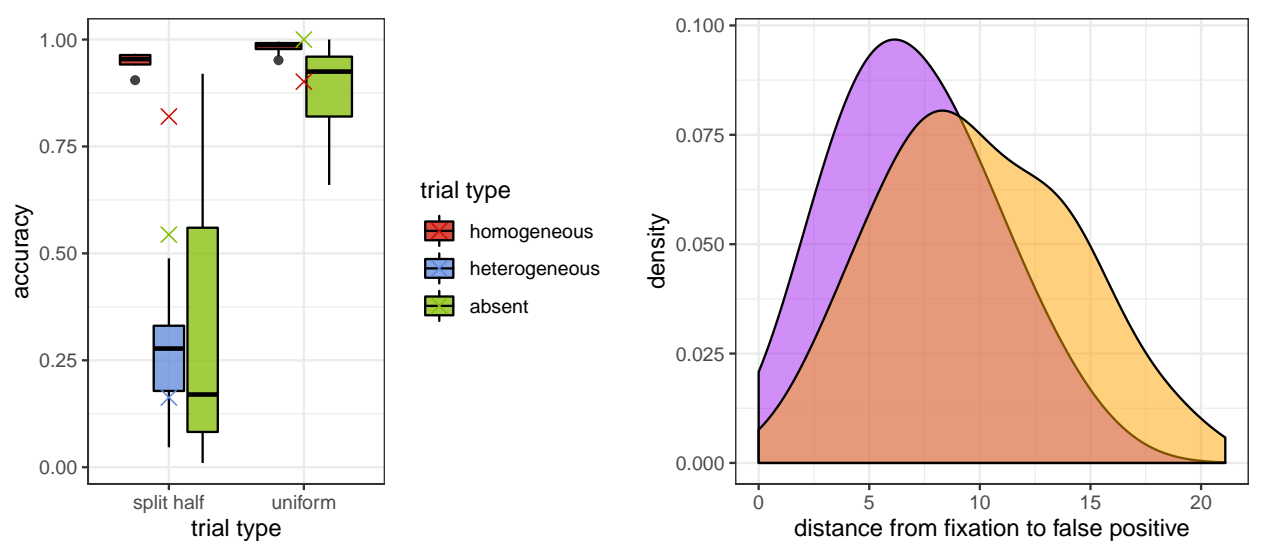

Fig 3. (left) Boxplot showing the distribution of accuracies for the detection experiment. The x's represent the performance of our detection model. (right) Distribution of false positives. Distances are measured from the fixation point to the location of the mouse click used to indicate an incorrect target present response. The purple distribution shows the empirical data, while the orange shows simulated data.

learning in human and animal brains, the type which we believe governs learning of task-specific visual search strategies. It makes no effort to learn a world model or reason about the task, and is driven purely by the local reward signal.

We carry out learning simulations with a population of 100 independent agents, each initialized with random network parameters and the same set of decision criteria. Initially, the agents tend to fixate both sides at random, but eventually, most of them converge to nearly-optimal policies (see Figure 44. In intermediate stages, however, a variety of strategies are observed, including sub-optimal ones.

We compare these against the range of strategies observed in human participants (Figure 5). We summarize each observer's strategy by computing how often they fixate in the heterogeneous side of the display. When this number is close to 1 , the observer is following a near-optimal strategy; when it is close to 0 , they are following an sub-optimal strategy. We do not necessarily expect a single population of agents to converge on the same distribution of strategies as our human experimental participants, since we expect humans to have other differences rather than representing a purely homogeneous population (discussed below). Instead, our focus is on the dynamics of the learning process and how they give rise to variability.

We plot the improvement in strategies across trials, by correlating each agent's performance at a particular time with performance in the future (Figure 5). Both humans and computational models show a tendency to improve over time (points above the $y=x$ line). For highly optimal agents (right side of the graph), the trend line dips below $y=x$ due to a ceiling effect (regression to the mean). Agents with less-optimal strategies in session $a$ show a wide variety of outcomes in session $b$, with only a weak correlation. This emphasizes the degree to which variability in the reward signal can determine the learning trajectory. Because these agents have not learned good strategies to find targets in the heterogeneous condition, they are dependent on finding it at random in order to get useful feedback.

Finally, we conduct a simulation intended to replicate the experiment done in Najemnik and Geisler (30). In this experiment, participants searched for a sine-wave grating pattern within a circular display. We use the same neural net architecture, learning algorithm and configuration as in the previous simulation, using the posterior probability of target presence at each location as the network input. We estimate the 

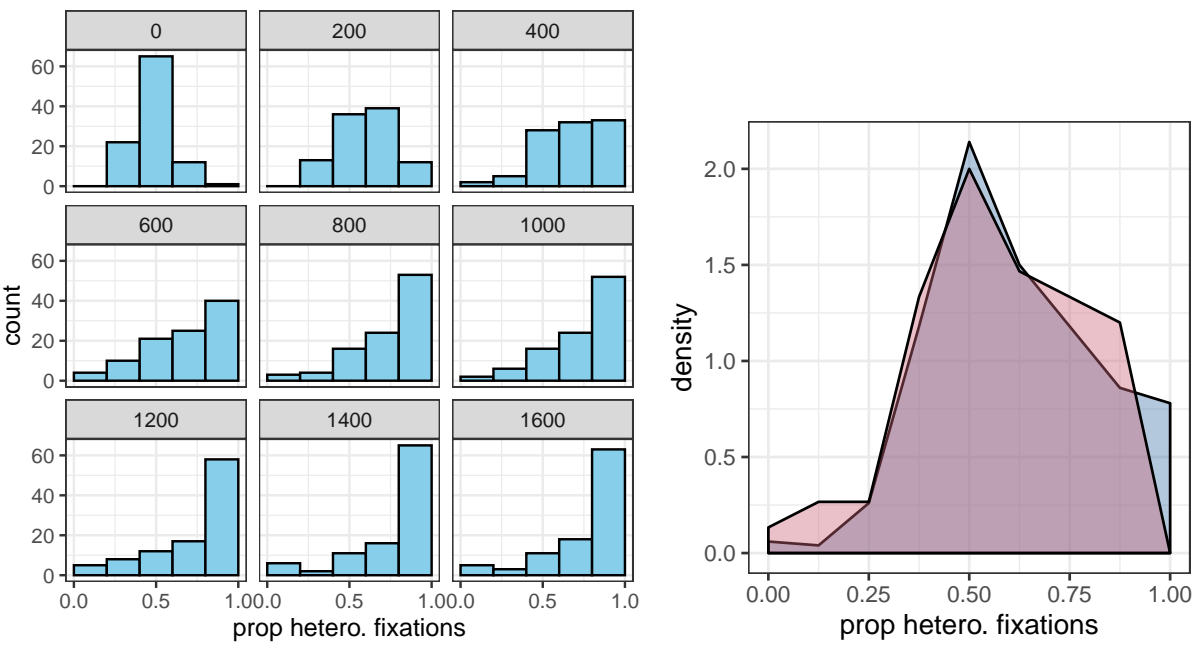

Fig 4. left: Evolution of the distribution of learned strategies over time, in a simulated reinforcement learning agent. $x$ axis shows proportion of fixations directed to the heterogeneous side of the display; $y$ axis shows number of agents. right: Comparison between the distribution of observed strategies in (red) human observers, and (blue) reinforcement agents (merging iter $=0,200,400$ and 600).
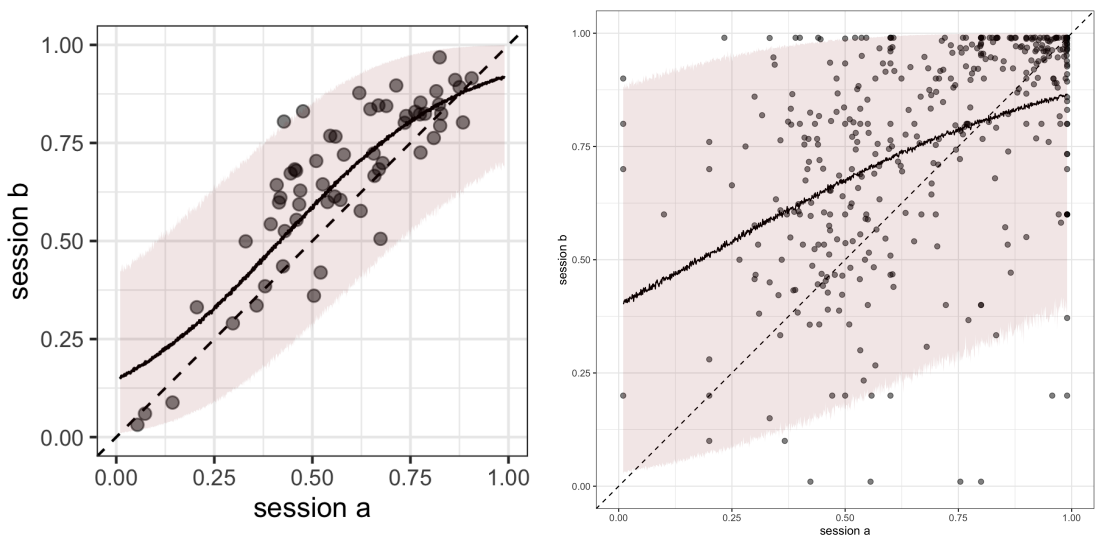

Fig 5. left: Distribution of observer strategies from (8). Each point represents the proportion of fixations directed to the heterogeneous side of the display by an observer in session a and session $b$. We can see that there is a wide range of individual differences, and most observers improve between sessions. right: The same plot, but with simulated data from our model. 

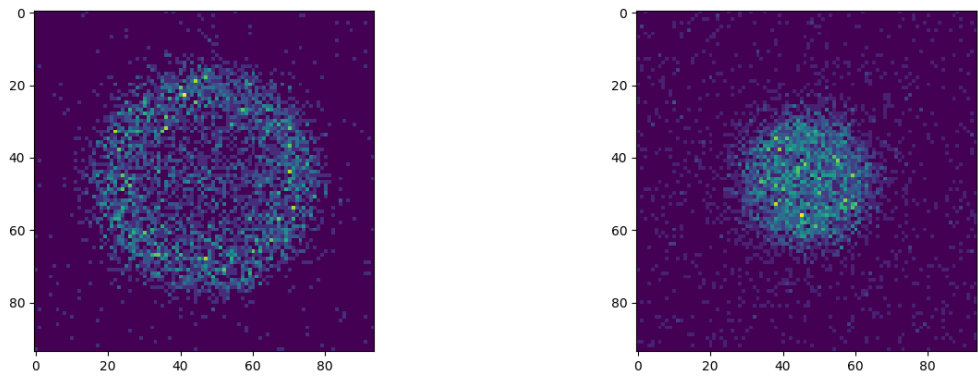

Fig 6. Fixation locations of two agents from a simulation of the experiment carried out by Najemnik and Geisler (30). The left agent has learned the "donut"-shaped pattern displayed by the optimal searcher, while the right agent has learned an sub-optimal central bias.

visual map parameters from Figure 2 of the original paper. In these scenes, the optimal model produces a "donut-shaped" pattern with a bias toward long saccades along the vertical axis. The competing MAP-search model, which saccades to the most likely target location, shows neither of these tendencies. Our model is capable of learning the "donut" pattern (Fig 6), though we have not observed the vertical bias.

However, the "donut" pattern is not the modal output after 10,000 iterations; rather, the majority of agents fail to learn useful policies at all, and some learn sub-optimal policies with central biases ("donut holes"). This is a consequence of the same properties that lead to variable solutions, slow convergence and the presence of sub-optimal solutions in the split-array stimuli. Simple policy gradient algorithms are reliant on the trial reward signal to indicate whether a given action is better or worse than average, but this signal has high variance (39). The variance is particularly high for the Najemnik and Geisler (30) task due to the small size of the target relative to the stimulus - even when following the optimal policy, finding the target is difficult.

If our algorithm is indeed a good model for learning in visual search tasks, we suggest two explanations for its difficulty in discovering good policies for the Najemnik and Geisler (30) task. One is that, since searching large, relatively uniform stimuli is the ecologically typical situation, humans spend much more time learning good policies for this kind of task than they do for the unfamiliar split-array task. Thus, even a high-variance learner may have time to converge. Second, the human learning mechanism may incorporate some variance-reduction mechanism, such as state-value estimation as in the Actor-Critic algorithm (Konda and Tsitsiklis). If this mechanism worked rapidly, we would expect much faster and more consistent learning on the split-array task, but perhaps it is relatively slow to accommodate to new situations.

\section{Conclusion}

When a participant comes into a laboratory to participate in a psychology experiment, we typically only observe a snapshot of their performance. The behavioral results from (8) presented here show that, while this visual search paradigm has reasonable test-retest reliability, there is significant learning from one week to the next. In the simulations presented above, we have demonstrated that these learning processes are capable of generating the wide range of individual differences among observers in the split-half visual search paradigm. Despite assuming a relatively homogeneous population of viewers (without differences in low-level visual acuity), we show that a 
cognitively plausible model of implicit learning gives rise to a variety of more and less effective search strategies. Some of these are relatively stable over the medium term despite their low task performance - indeed, due to the high variance of their reward signals, learners using poor strategies may get worse nearly as often as they get better. Nonetheless, learning outcomes eventually converge toward optimal behavior.

This view of variability can explain some seemingly conflicting observations which have been made about the learning process for visual search. On the one hand, searching behavior appears to incorporate some quite complex strategic behavior which is close to optimally optimal (30, 31); we suggest that, since reinforcement learning appears to converge over the long term, these behaviors are expected as the learning outcome for ecologically valid stimuli with characteristics similar to real-world images. Moreover, these strategies are implemented by a heuristic policy which does not use explicit, computationally intensive optimization or counterfactual reasoning. On the other, diversity in short and medium term learning outcomes explains not only the large variation between individuals on some laboratory tasks, but also the puzzling failure of these tasks to predict performance on one another (16). Rather than characterizing individuals as "more rational" or "less rational", and therefore expecting them to do better or worse on all tasks simultaneously, we should expect them to simultaneously try to learn strategies for each individual task, leading to independent learning outcomes biased mostly by variation in their initial policies and their early pattern of successes and failures within each task.

Of course, learning variability and variability in decision criteria are not the only factors which contribute to behavioral variation in search tasks. Some of these factors include differences in viewers' visual acuity, in their learning rates (the amount by which the policy changes after each trial), their reward functions (in particular, their speed-accuracy tradeoff), and their initial preferences for where to look. While these factors are important, we do not incorporate these into our model because we want to draw attention to the learning mechanism as an autonomous source of variability.

Typical experimental paradigms in psychology are designed to minimize variability between trials and between subjects, in order to make main effects of the controlled factors easier to detect (13). Thus, our understanding of human cognition is shaped by tasks in which short-term learning does not play a major role, since (as shown here), learning is a significant source of inter-trial variability, and therefore of lower test-retest reliability. But these results may understate the capacity of short-term implicit learning to affect human cognition. Rather than assume individual differences arise primarily from differences in personality or in low-level cognitive capacities like working memory, we must also consider differences due to divergence in learning trajectories.

\section{Materials and Methods}

Here, we first describe the how the empirical data was collected, and then outline the how the technical details behind our modelling results.

\section{Detection \& Search Experiment}

In this section, we describe our experimental replication of Nowakowska et al. (34). As well as carrying out this replication, we also collected data on a related detection task, which was used to estimate the visibility map component of our search model. Given the large amount of psychophysical data required to estimate the visibility map, the experiment was broken up into five sessions, and carried out over a week. Detection and search data was collected on each day. 


\section{Participants}

A total of nine participants took part in the study; one participant was removed from all analysis as they responded present to all target absent trials. In total, eight participants' data were included in the study ${ }^{1}$

\section{Apparatus}

The display was presented on a 17 -inch CRT monitor with a resolution of $1024 \times 768$. Stimuli were generated, presented and the data collected using MATLAB and the Psychophysics Toolbox (22). Eye movements were recorded using a desktop-mounted EyeLink 1000.

\section{Stimuli}

The stimuli consisted of black line segments on a uniformly grey background. Line segments formed a dense $24 \times 17$ grid, with each line segment of size $42 \times 42$ pixels. The distractor line segments' orientation was varied uniformly randomly - homogeneous line segments were varied up to $30^{\circ}$, and heterogeneous line segments up to $120^{\circ}$. The mean of distractor line segments' orientation was orthogonal to that of the target's. In both experiments, half of all trials consisted of a homogeneous array of line segments, and half of the trials a split-half stimulus. See Figure 1 for an example.

The target line segment was always angled $45^{\circ}$ to the right from vertical. As most targets against a heterogeneous array will not be detected, and hence be perceived as target absent by our participants, we decided to only include a small number of target absent trials in our detection experiment. There were 576 trials in total, $10 \%$ of which where target absent. The search experiment consisted of 96 trials, of which $50 \%$ had no target present.

\section{Procedure}

On arrival to the laboratory each participant was asked to read and sign a consent form. Participants were seated in a low-lit room, and were told that in the Detection Experiment they were to determine whether a line segment angled $45^{\circ}$ to the right was present in the stimulus. Participants were asked to respond as quickly and accurately as possible.

In the detection experiment, participants were asked to determine whether they saw the target line segment or not. Participants began by fixating on a cross displayed vertically in the centre of the screen, and horizontally between the 8th and 9th line segments (left), or between the 15th and 16th line segments (right). The position of the fixation cross was chosen randomly. When participants pressed space and the fixation was confirmed and a line segment stimulus was displayed for $200 \mathrm{~ms}$, after which the stimulus was replaced by an entirely grey background. Participants had to press the ' $\mathrm{f}$ ' key (did not see) or the ' $\mathrm{j}$ ' key (saw). If the participants pressed ' $\mathrm{j}$ ', indicated to have seen the target, an array or dots similar to the stimulus was displayed, and the participants were asked to indicate where the target was using the mouse.

If in either experiment the participants' response was incorrect (e.g. target indicated present, but was not present), a red screen was displayed and a warning sound played. Before both experiments, participants underwent a 14-point calibration sequence. Participants were re-calibrated between any blocks of trials if necessary.

\footnotetext{
${ }^{1}$ Due to a closure of the University of Aberdeen School of Psychology (COVID19), demographic data are not available at this time. Demographic data will be made available as soon as possible.
} 


\section{Data Processing}

To characterise the search strategy, we focus on saccades made during the target absent trials. Specifically, for each observer we calculate the proportion of trials in which the $i$ th fixation was on the heterogeneous side of the display, for $i=2,3,4,5$. Fixations landing close to the vertical midline are excluded. Trials resulting in a false positive are also excluded, as in these cases, the participant presumably fixates the part of the scene where they mistakenly believe they have seen the target, rather than continuing to apply their strategy for searching the scene when the target position is unclear.

\section{Psychophysical parameters}

Our visual search agents represent each object in the array by maintaining a posterior distribution over its orientation. Following Najemnik and Geisler (30), we use the results of the detection experiment to determine how reliable each update is at a given point, relative to the fixated location.

Because our focus is on the ways in which differences can arise from the learning algorithm, we estimate a single set of psychophysical parameters by aggregating the data from all participants. The aggregation approach also makes sure that we have enough data to obtain reliable estimates. Estimation of participant-specific visual parameters is left for future work.

To estimate the psychophysical parameters, we construct a formal probabilistic model of the detection experiment. We denote the orientation of each line segment by $\theta_{i}$, indexed by location $i$, and compute a posterior distribution $P\left(\theta_{i} \mid w_{i}\right)$ given noisy observations $w$. We assume that a single fixation yields an observation for each location in the display; however, the reliability of these fixations varies with their distance and angle to the fixation point. We model this by treating $w_{i}$ as a random variable, which is equal to the true orientation plus "internal noise". The variance of this noise

distribution is the main factor in determining how much of the scene is clearly visible given a fixation. In Appendix 0.1, we describe the generative process of the model in full.

Given the posterior over the orientation, the model computes a critical probability $p_{i}$ at each location, which is the estimated probability that this location contains a target (unconditioned on the rest of the scene). To make its final detection decision $d$, the model employs the Luce (softmax) decision rule (25). The decision depends on the $p_{i}$, along with two additional parameters. $a$ controls the observer's willingness to say that the target is absent, and $\lambda$ the "hardness" of the decision function (versus probability-matching behavior).

$$
d \sim \frac{p_{i, 1}^{\lambda}}{a^{\lambda}+\sum_{j=0}^{n} p_{j, 1}^{\lambda}}
$$

The model depends on free parameters which determine the internal noise variance as a function of distance and angle to the fixation point, as well $a$ and $\lambda$. Given these parameters, we can determine the likelihood that a participant reports that the target is located at any point in the stimulus array (whether or not that location is the true target position) or reports that the target is absent. Appendix 0.1 also explains how we optimize the parameters of the model by gradient descent on this likelihood function.

\section{Learning to saccade}

We model the visual search problem as a Markov decision problem to which reinforcement learning can be applied. At each timestep $t$, the model chooses a location $f_{t}$ to fixate, determined by its current search policy $\pi$ with parameters $r$ and its 
representation of the scene. It receives a noisy observation of the scene given this

location, and deterministically performs a posterior update of its representation. Then it makes a decision, again determined by its policy, of whether to halt the trial and deliver a response, or keep going. If it halts the trial, it receives a reward signal; if not, the timestep advances and it selects another fixation.

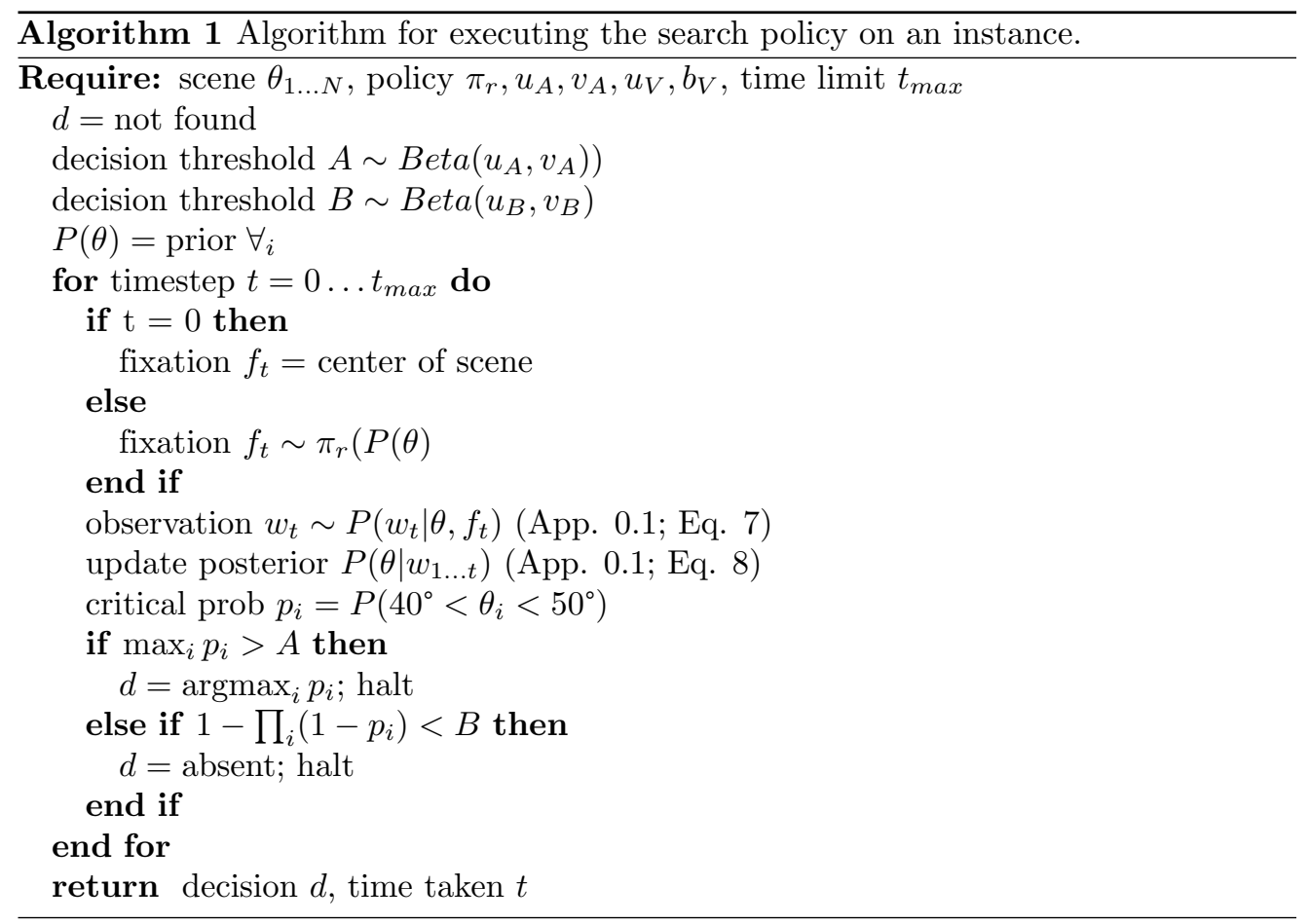

The model decides when and how to respond based on two thresholds, $A$ for positive decisions and $B$ for negative ones. If, after a posterior update, any critical probability $p_{i}>A$, the model responds that the target is present at $i^{*}=\operatorname{argmax}_{i} p_{i, t}$, the location with the highest probability of being the target. Otherwise, it computes the probability that the target is present anywhere in the scene:

$$
P(\text { target present })=1-\prod_{i}\left(1-p_{i}\right)
$$

If this probability is less than $B$, the model reports that the target is absent. If neither threshold is met, the search continues until the maximum number of iterations, then terminates without a response.

The thresholds $A$ and $B$ are sampled from independent Beta distributions at the start of each trial ${ }^{2}$ The Beta distribution has support on the interval $[0,1]$ and is controlled by two parameters $u, v$ which control the location of the mean $\left(\frac{v}{u+v}\right)$ and the concentration (larger parameter values yield a narrower distribution). We treat these distributions as part of our policy $\pi$, and the values of $A$ and $B$ as actions. Thus, in Equation 4, $\log \pi$ is augmented with the $\log$-probability of $A, \log P\left(A ; u_{A}, v_{A}\right)$ and likewise for $B$. The update equation alters the parameters so that the distribution moves towards and becomes concentrated around thresholds associated with successful 
trials, but moves away from unsuccessful ones. We use Tensorflow Probability (11) to compute the appropriate gradients.

The saccade selection network $\pi$ takes as input a representation of the posterior $P\left(\theta_{i} \mid w_{i, 1 \ldots t}\right)$ discretized into $5^{\circ}$ bins. This representation is augmented with the index of the location $i$, represented as a 1-hot, so that the model can learn location-specific preferences such as central biases. The model is a four-layer neural network. It generates a latent representation of the posterior at each location, performs a $3 \times 3$ spatial convolution over these, then a second $3 \times 3$ spatial convolution. All these representations are concatenated, providing a map with the same spatial dimensions as the image, containing both local (item-specific) and larger-scale (item neighborhood) representations at each point. These features are passed through a matrix multiplication and softmax to derive the probability of fixating each location. We denote the entire network as policy $\pi$ with parameters $r$.

We apply a relatively simple reward function $R$. We assume that each timestep of searching carries a cost. We also assume that participants earn a reward when they are correct, but not when they are wrong. (Participants in the experiment knew whether they are right or wrong due to the flashing red feedback screen displayed when they made a mistake.) In full, the reward for decision $d$, at timestep $t$, with true scene $\Theta$ is:

$$
R(d, t, \Theta)= \begin{cases}3-t & \text { decision correct } \\ -t & \text { otherwise }\end{cases}
$$

Learning is carried out via policy gradient (44). At each learning iteration, we execute the current policy $\pi$ on a batch of random stimuli, recording both the reward and the gradient of the actions taken with respect to the network parameters. We compute the difference between the reward value of the current policy, and the average reward; we use this to reweight the gradient and perform a gradient update of our parameters $r$ :

$$
\Delta r=\alpha \nabla_{r} \log \pi_{r}(P(\theta))\left(R\left(\pi_{r}(P(\theta))-E[R]\right)\right.
$$

Under this update rule, when the actions just taken perform better than average, the parameters are changed to make them more probable under the updated policy; conversely, when they are worse, the parameters are changed to make them less probable.

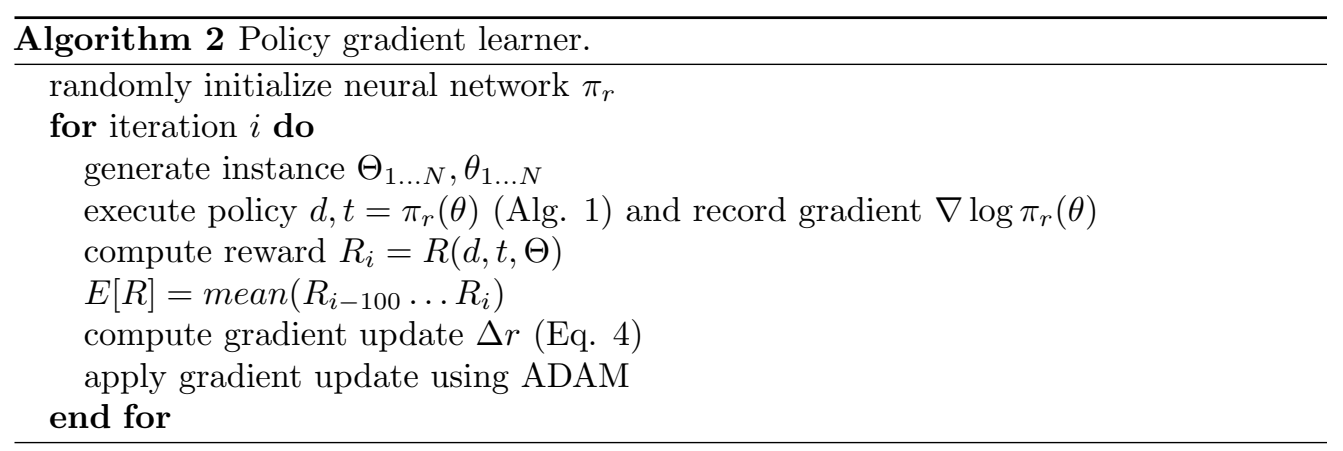

We initialize the network $\pi$ using the default CNN parameter initializers in Keras (6), but manually initialize the $u$ and $v$ parameters which control the decision thresholds to control the initial mean values of $A$ and $B$. Our main results set both these means to .5 , through setting $u=20, v=20$. (We tuned the magnitude of these parameters manually so that sampling would yield a reasonable amount of local exploration close to the initial mean threshold.) 
In the experiments below, we train on randomly-generated stimuli, half with the target present and half with it absent. When present, the target may appear on either side. The parameter learning rates are set using Adam (21) with default parameters. The baseline value estimate $E[R]$ is set to the moving average of the last 100 batches. We process 4800 stimuli per training run.

\subsection{Simulating Najemnik and Geisler (30)}

In our second simulation, we simulate the search experiment from Najemnik and Geisler (30). In that experiment, participants searched a $15^{\circ}$ circular noise region for a sine wave grating pattern approximately $\frac{1}{6}^{\circ}$ in width.

We represent this type of stimulus as a rectangular array of possible target positions, $94 \times 94$ in size. Each element in the central circular region (with radius 47 ) contains values +.5 (target) or -.5 (non-target) plus internal noise; the corners are blank. At each position, we represent the posterior probability of target presence given the previous fixations, applying equations 4 and 5 from the original paper. We use the approximate visual map parameters from the paper, estimated by measuring the extreme points of the isobars in Figure 2, assuming the contours are elliptical, and then interpolating to obtain the intermediate values. Thus, we believe our posterior values (the inputs to the search procedure) are comparable to those used in the original work.

We use the same network architecture and learning algorithm as for the split-half simulation; since it is convolutional, it scales naturally to the larger stimulus. We use the same learning algorithm, training on 10,000 random stimuli per agent. Because the stimuli are much larger than those in the split-half study, the target is quite difficult to find. Thus, we set the initial threshold $A$ to a very low value, .005, since, as previously discussed, the model relies on finding the target by accident to bootstrap its early learning behavior. Because the variance of the reward signal goes up as the length of the searches increases, we also set the training time limit to 3 fixations per trial.

In a population of 100 agents, only two agents learn "donut"-shaped patterns. No agents exhibit significant vertical bias, as displayed by the optimal models. Five agents learn central biases, fixating repeatedly near the center of the display, despite this yielding no new information because the initial fixation location is at the center. The remaining agents learn basically unstructured policies which fixate randomly throughout the stimulus.

\section{Supporting information}

\section{Appendix: estimation of the visual map}




\section{Appendix A: Estimation of detection parameters}

As previously stated, the model's mental representation of the image abstracts away from low-level visual detail, maintaining a distribution over the item at each point in the grid. Formally, let the grid consist of locations $i \in 0 \ldots N$, each of which has a "true orientation" $\Theta_{i}$. In our experiment, $\Theta_{i}=45^{\circ}$ at the target location and $-45^{\circ}$

everywhere else. We designate a "heterogeneous" region (either the left or the right side of the array); let $h_{i}=1$ when location $i$ is within this region and 0 otherwise.

A particular stimulus is sampled by adding "external noise" $\nu_{i}^{E}$ (sampled once per stimulus) to the $\Theta$ values. In our experiment, there is no noise at the target, relatively little noise in the homogeneous region and a larger amount of noise in the heterogeneous region. (The noise distributions are, however, selected so that no distractor can overlap the target orientation.)

$$
\begin{array}{llll} 
& & & \nu_{i}^{E} \sim \\
0 & \text { if } \Theta_{i}=45^{\circ} & \\
x x & \text { if } h_{i}=0 \\
x x & \text { if } h_{i}=1
\end{array}
$$

Denote the angle at $i$, as it actually appears in the stimulus, as $\theta_{i}=\Theta_{i}+\nu_{i}^{E}$. Our model represents the probability distribution over $\theta_{i}$ at each location $i$. This distribution evolves as the model makes (unreliable) observations. At each observation time $t$, the model observes orientation, $w_{i, t}$, for each item in the display; $w_{i, t} \sim \theta_{i}+\nu_{i, t}^{I}$, where $\nu_{i, t}^{I}$ is "internal noise", which is randomly sampled fixation by fixation. The amount of noise at location $i$ depends on the position of location $i$ relative to the fixation point $f_{t}$, and whether $i$ is within the hard region or not. In particular, we assume that $\nu^{I}$ has 0 mean, but a location-dependent variance $\sigma$ (determined by a function $H$, which is described below):

$$
\begin{aligned}
\nu_{i, t}^{I} & \sim \mathcal{N}\left(0, \sigma_{i, t}\right) \\
\sigma_{i, t} & =H\left(i, f_{t}\right)
\end{aligned}
$$

The model's scene representation at time $t$ is then $P\left(\theta_{i} \mid w_{1 \ldots t}\right)$ for each location $i ; w$ in turn depends on $O, h$ and the internal and external noise. The posterior update is:

$$
P\left(\theta_{i} \mid w_{1 \ldots t}\right) \propto P\left(\theta_{i} \mid w 1 \ldots t-1\right) \times \mathcal{N}\left(0, \sigma_{i, t}\right)
$$

If we choose a Gaussian prior, the posterior will also be Gaussian. In particular, if $P\left(\theta_{i} \mid w 1 \ldots t-1\right)$ has distribution $\mathcal{N}\left(\mu_{t-1}, \sigma_{t-1}\right)$, then the posterior has distribution:

$$
\begin{aligned}
P\left(\theta_{i} \mid w_{1 \ldots t}\right) & =\mathcal{N}\left(\mu_{t}, \sigma_{t}\right) \\
\mu_{t} & =\frac{\sigma_{i, t}, t_{t-1}}{\sigma_{i, t}+\sigma_{t-1}} \\
\sigma_{t} & =\frac{1}{\frac{1}{\sigma_{t-1}}+\frac{1}{\sigma_{i, t}}}
\end{aligned}
$$

So far, this approach is exactly parallel to that of Najemnik and Geisler (30), except that we have decided to maintain a distribution over the orientation $\theta_{i}$, rather than directly over the probability that an item is the target, $P\left(\theta_{i}=45^{\circ}\right)$. The latter representation would deny our control policy access to information about the image other than the likely presence or absence of a target - in particular, it could not determine whether a given distractor is likely to be part of the easy or hard region, even though this is easily guessed given an estimate of its orientation. 
Our choice of representation does require us to state explicitly how the viewer converts the orientation probability into the target probability in order to make decisions. We assume that this is done by integrating the posterior over a small "critical region" around the target. The use of an integral (rather than the point value of the $\mathrm{PDF}$ ) is computationally convenient for the neural network learner described in the next section. That learner is more stable when provided with discrete features, so in that section, we approximate the posterior with a discrete distribution by binning. Since our critical probability is an integral, it can be easily computed by summing bins.

We set the width of the critical region to $\delta=5^{\circ}$ (note again that no distractor orientation can fall within this range). We denote the critical probability that the target lies within it as $c_{i, t}$ where $c_{i, t}=P\left(40 \leq \theta_{i} \leq 50\right)=c d f\left(P\left(\theta_{i} \mid w_{i, 1 \ldots t}\right), 50\right)-c d f\left(P\left(\theta_{i} \mid w_{i, \ldots t}\right), 40\right)$.

Given these critical probabilities, we are equipped to model the outcomes of the detection experiment. (Recall that this experiment involves a single fixation on a pre-determined location, after which the participant makes a forced choice between "target present" and "target absent".) We assume that the agent has a parameter indicating their willingness to say the target is absent, which we denote $a$. It is difficult to set an a priori value for $a$, because it is not clear what utility function for false positives versus false negatives we should assume, nor what prior we should put on target presence in the scene, so we will treat $a$ as a free parameter to estimate from the data.

We use a soft, rather than a hard, decision function (that is, we assume participants might make decisions either by probability matching or by maximizing). There are two reasons for this: first, it is mathematically convenient, since the soft choice rule is differentiable while the maximum function is not. Second, probability matching is a commonly observed behavior in psychological experiments. We model the greediness of the observer's decisions via a parameter $\lambda$ which determines how they choose among options based on their probabilities. Specifically, the decision $d$ is sampled from:

$$
d \sim \frac{p_{i, 1}^{\lambda}}{a^{\lambda}+\sum_{j=0}^{n} p_{j, 1}^{\lambda}}
$$

$\lambda=1$ yields pure probability matching; $\lambda=\infty$ yields pure maximization (in practice, this holds for finite $\lambda>>1$ ). $\lambda<1$ smooths towards uniformity.

To summarize, the decision process is:

- Make a single observation $w_{0 . . . n, 1,1}$ at a pre-selected fixation point $f_{1}$

- Compute the posterior distribution on the orientation at each point, $P\left(\theta_{i} \mid w_{i, 1}\right)$

- Compute the probability that the orientation lies within the critical region for each point, $p_{i, 1}=P\left(\theta^{T}-\delta<\theta_{i}<\theta^{T}+\delta \mid w_{i, 1}\right)$

- Sample the decision $d$ from the renormalized posterior, plus $a$ as the term indicating the strength of the agent's preference to respond "absent"

As previously stated, we assume that the $\sigma$ noise variance terms are related to one another via a function $H$, ensuring that $\sigma$ varies smoothly across the visual field. In particular, we use a linear model in which $H\left(i, f_{i}\right)=V \cdot F\left(i, f_{i}\right)$, where $V$ is an unknown parameter vector and $F\left(i, f_{i}\right)$ is a feature vector for location $i$ relative to fixation point $f_{i}$. We find good results using a radial basis function representation for $F$ (4), in which distances and angles are represented non-parametrically, in terms of the responses of a collection of fixed basis functions $\phi_{j}$. We use the normal density with mean $j$ as our basis. For distance, $\phi_{j}\left(i, f_{i}\right)=N\left(\left|i, f_{i}\right|_{2} ; j, 1\right)$. We use 10 equally spaced 
points $j$ between 0 and 20 (roughly equal to the longest distances seen in the detection experiment). For angles, we use 5 points spaced between 0 and 90 degrees, and set the normal variance to 10 . Using this feature representation ensures that the variance $\sigma$ changes smoothly as a function of distance and angle, while also allowing us to represent non-linear trends.

Thus, the parameters of the model which must be estimated are $V, a$ and $\lambda$. Our objective in setting these parameters is to maximize the probability of the observed decision on each trial, conditioned on the stimulus and the fixation location. This decision depends on the noisy observations $w$, which we do not have access to, so we integrate them out:

$$
\sum_{\text {trial } j} \log P\left(d_{j} \mid \theta_{1 \ldots N}^{j}, f_{1}^{j}\right)=\sum_{\text {trial } j} \log \int_{w_{1 \ldots N}} P\left(w_{1 \ldots N} \mid \theta_{1 \ldots N}, f_{1}^{j}\right) P\left(d_{j} \mid w_{1 \ldots N}\right) d w
$$

We approximate the integral over $w$ by sampling (2). Using the Tensorflow Probability package (11), this sampling operation can be incorporated into a standard Tensorflow training graph, yielding an estimate of the gradient of the likelihood function. We use the Adam optimizer (21) to stochastically maximize the likelihood as a function of the parameters. 


\section{Acknowledgments}

This work was supported by The Rank Prize Funds optoelectronics vacation studentship

(B. Lonnqvist) and ESRC (ES/S016120/1) grant to A. Hunt and A. Clarke.

\section{References}

1. Ba, J., Mnih, V., and Kavukcuoglu, K. (2014). Multiple object recognition with visual attention. arXiv preprint arXiv:1412.7755.

2. Bishop, C. M. (2006). Pattern recognition and machine learning. Springer.

3. Blakemore, C. and Van Sluyters, R. C. (1975). Innate and environmental factors in the development of the kitten's visual cortex. The Journal of physiology, 248(3):663-716.

4. Buhmann, M. D. (2003). Radial basis functions: theory and implementations, volume 12. Cambridge university press.

5. Bylinskii, Z., Judd, T., Borji, A., Itti, L., Durand, F., Oliva, A., and Torralba, A. (2016). Mit saliency benchmark. http://saliency.mit.edu/.

6. Chollet, F. (2015). Keras. https://github.com/fchollet/keras.

7. Clarke, A. D., Green, P., Chantler, M. J., and Hunt, A. R. (2016). Human search for a target on a textured background is consistent with a stochastic model. Journal of vision, 16(7):4-4.

8. Clarke, A. D., Irons, J., James, W., Leber, A. B., and Hunt, A. R. (2020). Stable individual differences in strategies within, but not between, visual search tasks. Quarterly Journal of Experimental Psychology.

9. Clarke, A. D., Nowakowska, A., and Hunt, A. R. (2019). Seeing beyond salience and guidance: the role of bias and decision in visual search. Vision, 3(3):46.

10. Daw, N. D., Niv, Y., and Dayan, P. (2005). Uncertainty-based competition between prefrontal and dorsolateral striatal systems for behavioral control. Nature neuroscience, 8(12):1704-1711.

11. Dillon, J. V., Langmore, I., Tran, D., Brevdo, E., Vasudevan, S., Moore, D., Patton, B., Alemi, A., Hoffman, M., and Saurous, R. A. (2017). Tensorflow distributions. arXiv preprint arXiv:1711.10604.

12. Gläscher, J., Daw, N., Dayan, P., and O’Doherty, J. P. (2010). States versus rewards: dissociable neural prediction error signals underlying model-based and model-free reinforcement learning. Neuron, 66(4):585-595.

13. Hedge, C., Powell, G., and Sumner, P. (2018). The reliability paradox: Why robust cognitive tasks do not produce reliable individual differences. Behavior Research Methods, 50(3):1166-1186.

14. Imbert, M. and Buisseret, P. (1975). Receptive field characteristics and plastic properties of visual cortical cells in kittens reared with or without visual experience. Experimental Brain Research, 22(1):25-36.

15. Irons, J. L. and Leber, A. B. (2016a). Choosing attentional control settings in a dynamically changing environment. Attention, Perception, \&3 Psychophysics, 78(7):2031-2048. 
16. Irons, J. L. and Leber, A. B. (2016b). Choosing attentional control settings in a dynamically changing environment. Attention, Perception, 83 Psychophysics, pages $1-18$.

17. Irons, J. L. and Leber, A. B. (2018). Characterizing individual variation in the strategic use of attentional control. Journal of Experimental Psychology: Human Perception and Performance, 44(10):1637.

18. Itti, L. and Koch, C. (2000). A saliency-based search mechanism for overt and covert shifts of visual attention. Vision research, 40(10-12):1489-1506.

19. Itti, L., Koch, C., and Niebur, E. (1998). A model of saliency-based visual attention for rapid scene analysis. Pattern Analysis and Machine Intelligence, IEEE Transactions on, 20(11):1254-1259.

20. Kahneman, D. (2011). Thinking, fast and slow. Macmillan.

21. Kingma, D. P. and Ba, J. (2014). Adam: A method for stochastic optimization. arXiv preprint arXiv:1412.6980.

22. Kleiner, M., Brainard, D., Pelli, D., Ingling, A., Murray, R., and Broussard, C. (2007). What's new in psychtoolbox-3. Perception, 36(14):1-16.

Konda and Tsitsiklis. Konda, V. R. and Tsitsiklis, J. N. Actor-critic algorithms. pages 1008-1014.

24. LeCun, Y., Kavukcuoglu, K., and Farabet, C. (2010). Convolutional networks and applications in vision. In Proceedings of 2010 IEEE international symposium on circuits and systems, pages 253-256. IEEE.

25. Luce, R. D. and Green, D. M. (1974). Detection, discrimination, and recognition. Handbook of perception, 2:299-342.

26. Ludvig, E. A., Bellemare, M. G., and Pearson, K. G. (2011). A primer on reinforcement learning in the brain: Psychological, computational, and neural perspectives. In Computational neuroscience for advancing artificial intelligence: Models, methods and applications, pages 111-144. IGI Global.

27. Mnih, V., Heess, N., Graves, A., et al. (2014). Recurrent models of visual attention. In Advances in Neural Information Processing Systems, pages 2204-2212.

28. Mnih, V., Kavukcuoglu, K., Silver, D., Rusu, A. A., Veness, J., Bellemare, M. G., Graves, A., Riedmiller, M., Fidjeland, A. K., Ostrovski, G., et al. (2015). Human-level control through deep reinforcement learning. Nature, 518(7540):529.

29. Morvan, C. and Maloney, L. T. (2012). Human visual search does not maximize the post-saccadic probability of identifying targets. PLoS computational biology, $8(2)$.

30. Najemnik, J. and Geisler, W. S. (2008). Eye movement statistics in humans are consistent with an optimal search strategy. Journal of Vision, 8(3):4-4.

31. Najemnik, J. and Geisler, W. S. (2009). Simple summation rule for optimal fixation selection in visual search. Vision research, 49(10):1286-1294.

32. Navalpakkam, V. and Itti, L. (2005). Modeling the influence of task on attention. Vision research, 45(2):205-231. 
33. Navalpakkam, V. and Itti, L. (2007). Search goal tunes visual features optimally. Neuron, 53(4):605-617.

34. Nowakowska, A., Clarke, A. D., and Hunt, A. R. (2017). Human visual search behaviour is far from ideal. Proceedings of the Royal Society B: Biological Sciences, 284(1849):20162767.

35. Pavlov, I. (1927). Conditioned Reflexes: An Investigation of the Physiological Activity of the Cerebral Cortex" Translated by GV Anrep. Oxford University Press LONDON.

36. Posner, M. I., Snyder, C. R., and Davidson, B. J. (1980). Attention and the detection of signals. Journal of experimental psychology: General, 109(2):160.

37. Shah, A. (2012). Psychological and neuroscientific connections with reinforcement learning. In Reinforcement Learning, pages 507-537. Springer.

38. Silver, D., Huang, A., Maddison, C. J., Guez, A., Sifre, L., Van Den Driessche, G., Schrittwieser, J., Antonoglou, I., Panneershelvam, V., Lanctot, M., et al. (2016). Mastering the game of go with deep neural networks and tree search. nature, 529(7587):484.

39. Sutton, R. S. and Barto, A. G. (1998). Reinforcement learning: An introduction, volume 1. MIT press Cambridge.

40. Sutton, R. S. and Barto, A. G. (2018). Reinforcement learning: An introduction. MIT press.

41. Treisman, A. M. and Gelade, G. (1980). A feature-integration theory of attention. Cognitive psychology, 12(1):97-136.

42. Vasilyev, A. (2018). Optimal control of eye movements during visual search. IEEE Transactions on Cognitive and Developmental Systems, 11(4):548-559.

43. Vig, E., Dorr, M., and Cox, D. (2014). Large-scale optimization of hierarchical features for saliency prediction in natural images. In Proceedings of the IEEE Conference on Computer Vision and Pattern Recognition, pages 2798-2805.

44. Williams, R. J. (1992). Simple statistical gradient-following algorithms for connectionist reinforcement learning. Machine learning, 8(3-4):229-256.

45. Wolfe, J. M. (1994). Guided search 2.0: A revised model of visual search. Psychonomic Bulletin \&3 Review, 1:202-238.

46. Wolfe, J. M. (2012). Visual search. In Todd, P., Holls, T., and Robbins, T., editors, Cognitive Search: Evolution, Algorithms and the Brain, pages 159-175. MIT Press, Cambridge, MA, USA.

47. Wolfe, J. M. and Gray, W. (2007). Guided search 4.0. Integrated models of cognitive systems, pages 99-119.

48. Zelinsky, G. J. (2008). A theory of eye movements during target acquisition. Psychological review, 115(4):787. 